\title{
Determinación del tratamiento de los agentes causales de la caspa (dermatitis escamosa) en la fibra de vicuña (Vicugna vicugna)
}

\author{
Determination of the treatment of the causal agents \\ of the dandruff (scaly dermatitis) in the vicuña fiber (Vicugna vicugna)
}

\author{
Luis Chacón Navarro*
}

http://dx.doi.org/10.21503/CienciayDesarrollo.2011.v13.03

\section{RESUMEN}

Se ha reportado la presencia de pequeñas descamaciones (caspa o tejido epitelial muerto de la piel) en la fibra de la vicuña a nivel nacional, lo que está ocasionando pérdidas económicas realmente considerables en el productor altoandino, que no cuenta con una solución inmediata para controlarla, curarla y sobre todo prevenirla. Entre las causas posibles de esta enfermedad podemos considerar:

Factores metabólicos: Situaciones de estrés, desnutrición y avitaminosis son factores que influyen sobre la calidad del tejido epitelial de la piel de la vicuña.

Factores externos: Condición sanitaria del animal (parásitos externos, cómo ácaros) y condiciones medioambientales (radiación solar, temperatura, etc.).

El objetivo principal de este trabajo es determinar el tratamiento del agente causal posible, realizando las observaciones histológicas y biológicas directas en la caspa de los animales y en los vellones de fibra de vicuña.

La experiencia se ha realizado aprovechando el tercer y cuarto chaco (años 2009 y 2010) de la población de animales que se encuentran en el corral perenne del Comité de Conservación y Uso Sostenible de la Vicuña del CIPTT Tullpacancha, Universidad Alas Peruanas, en el distrito de Locroja, provincia de Churcampa, Huancavelica, Perú.

De las 703 vicuñas capturadas en el chaco 2009 ( 18 y19 de julio de 2009), se evaluaron 74 vicuñas capturadas que presentaban anomalías dermatológicas (caspa), las que representaban el 10,53\% de la población estimada.

Realizando una labor de manejo productivo que incluía la aplicación del tratamiento preventivo veterinario con ivermectina y correctores nutricionales (vitaminas A,D,E y K) a los animales afectados en la campaña 2009, se llegó a comprobar en la campaña de 2010 la limpieza de la fibra de 15 animales afectados con caspa. Del resto de animales afectados (59 vicuñas) no existen antecedentes, porque no fueron capturados en el chaco 2010, pero se espera identificarlos en el chaco 2011.

Palabras clave: ivermectina, hematovit.

\section{ABSTRACT}

It has been reported the presence of small flaking (dandruff, dead skin tissue of the skin) in the vicuña wool nationwide, this is really causing considerable economic losses in high-Andean producer, added to that the inability to provide an immediate solution regarding how to control, cure and above all to prevent it. Are considered possible causes:

* Zootecnista, administrador del Centro de Investigación, Producción y Transferencia Tecnológica (CIPTT ) y responsable del corral perenne del Comité de Conservación y Uso Sostenible de Camélidos Silvestres de Tullpacancha (UAP), distrito de Locroja, provincia de Churcampa, Región Huancavelica. (e-mail: chacon27@hotmail.com) 
Metabolic Factors: Malnutrition and vitamin deficiencies affect the quality of the epithelial tissue of the skin of the vicuña.

External Factors: Animal health condition (external parasites: mites, etc.), environment (solar radiation, temperature, etc.).

The main objective of this study is to determine the causal agent by their histological and microbiological the problem of dandruff in vicuña wool fleeces and determine the level of prevalence of the "dandruff" on the population of animals found on poultry perennial Conservation Committee and Sustainable Use of Vicuña Tullpacancha CIPTT UAP of the 703 captured del Corral vicuñas Perennial of the Committee for Conservation and Sustainable Use of Vicuña Tullpacancha Research Center, Production and Transfer of Technology Tullpacancha-UAP, located in the district and province Churcampa Locroja belonging to the days Huancavelica Region July 18-19, 2009 - III Chaco 200974 vicuñas captured evaluated that presented anomalies and pathological skin (dandruff), being this the $10,53 \%$ of the estimated population.

Running the work of handling productive, applying the extent of preventive treatment veterinarian: Ivermectin and correctors nutritional, the affected animals are brings me to clean the fiber 15 affected animals with dandruff.

Key words: ivermectina, hematovit.

\section{INTRODUCCIÓN}

\section{Antecedentes de estudio}

El problema fue detectado estos últimos años, no existiendo antecedentes estadísticos, y menos bibliográficos, relacionados con el tema. Aprovechando el traslado de las vicuñas de la comunidad campesina de Lucanas al CIPTT Tullpacancha - UAP en el mes de noviembre del 2006, se evaluaron 500 vicuñas para determinar la presencia de la dermatitis escamosa (caspa), identificándose a 4 animales afectados, de los que se procedió a realizar los análisis microbiológicos para determinar el agente causante. Los resultados fueron los siguientes

1. Análisis microbiológico realizado en Laboratorio Central de la Escuela de Medicina Veterinaria de la UAP

Se procedió a la evaluación microbiológica de los animales con presencia de caspa (dermatitis escamosa); Convenio UAP-Comunidad de Lucanas.
Responsable: Blgo. Mblgo. Deyli Días Lezama (Jefe del Laboratorio Central de la UAP).

Asunto: Informe № 013 de la Escuela de Medicina Veterinaria de la UAP - Laboratorio Central- Dic. 2006.

Resultados del análisis microbiológico de la caspa en las vicuñas de Pampas Galeras:

- Presencia de ácaros: negativo.

- Recomendaciones: realizar nuevos análisis y considerar la calidad de pastos.

2. Trabajo de investigación: Estudio de la capacidad de biomasa accesible del CIPTT Tullpacancha UAP, en condiciones climáticas normales, para determinar la densidad poblacional de vicuñas

Conclusión: Se observa una biomasa forrajera accesible (B.F.A) en el CIPTT Tullpacancha UAP anual (con proyecciones conservadoras) de 3614 TM de pasto nativo y de un alto valor nutritivo, tomando como punto de comparación 
a la alfalfa (Medicago sativa). De aquí se deduce la existencia de una cantidad de $9900 \mathrm{~kg}$ de pasto óptimo para consumo diario (considerando un corte anual). Si tenemos en cuenta que cada vicuña consume diariamente $1300 \mathrm{~g}$ de forraje, se obtiene un promedio de consumo total de $4850 \mathrm{~kg}$. Elotro 50,0\% (4850 kg) correspondería a perdidas de forrajes por diversas causas, que proveería alimento suficiente para la crianza en semicautiverio de 3800 cabezas en condiciones normales.

\section{Objetivos}

\section{Objetivo general}

- Determinar el agente causal utilizando una evaluación histológica y microbiológica del problema de la caspa que vienen presentando las vicuñas del Comité de Conservación y Uso Sostenible de la Vicuña del CIPTT Tullpacancha - UAP.

\section{Objetivos especificos}

- Determinar el nivel de prevalencia de la caspa en la población de vicuñas.

- Determinar las medidas preventivas sanitarias para evitar la prevalencia de la caspa en la población de vicuñas del CIPTT Tullpacancha - UAP.

- Caracterizar la etiología de la caspa buscando definir a qué edad aparece, así como qué sexo y qué zona del cuerpo son los más afectados.

\section{Hipótesis}

La presencia de caspa (dermatitis escamosa) en las vicuñas del Corral Perenne del Comité de Conservación y Uso Sostenible de la Vicuña Tullpacancha - UAP (Centro de Investigaciones, Producción y Transferencia Tecnológica) sería causada por:
Factores metabólicos: Deficiencia nutricional.

Factores externos: Manejo, condición sanitaria del animal (parásitos externos, cómo ácaros) y condiciones medioambientales (radiación solar, temperatura, etc.).

\section{Fundamentos}

El Perú posee el 54,95\% aproximadamente de la población de vicuñas de nuestro continente, lo que la convierte en un factor de especial importancia socioeconómica para las zonas más empobrecidas de nuestro país, puesto que no solo provee ingresos económicos obtenidos de la comercialización de la fibra en los mercados textiles del continente europeo, sino que además genera puestos de trabajo que son de vital importancia para el desarrollo de las zonas empobrecidas de la región altoandina que cuentan con este recurso animal.

\section{Tabla 1}

\begin{tabular}{l|r|r|c}
\multicolumn{1}{c|}{ PAÍs } & POBLACIÓN & \multicolumn{1}{c|}{$\%$} & $\begin{array}{c}\text { CENSO/ } \\
\text { PROYECCIÓN }\end{array}$ \\
\hline Perú & 188327 & 54,95 & 2006 \\
\hline Argentina & 72678 & 21,21 & 2006 \\
\hline Bolivia & 62869 & 18,34 & 2006 \\
Chile & 16170 & 4,72 & 2006 \\
\hline Ecuador & 2683 & 0,78 & 2006 \\
\hline TOTAL & 324727 & & \\
\hline
\end{tabular}

Fuente: Censo Nacional 2000/Proyecciones del CONACS.

Es preocupante el problema que se viene suscitando en el vellón de la fibra de la vicuña. Nos referimos a la aparición de pequeñas descamaciones (caspa) del epitelio en diferentes partes del cuerpo, e incluso, a veces, en todo el cuerpo del animal, agravada por el hecho de que las causas de este mal aún se desconocen. Este problema dificulta el procesamiento textil e incrementa 
grandemente las pérdidas económicas en los productores vicuñeros altoandinos, toda vez que no pueden esquilar los animales que presentan la caspa.

A diferencia de otras enfermedades, la caspa no presenta sintomatología, por lo que es detectada recién al momento de la manipulación de los animales para su clasificación en el proceso de esquilar. Según lo manifestado, esta enfermedad es muy peligrosa para la economía de los productores vicuñeros por la incapacidad de prever una solución inmediata o a mediano plazo para controlarla y, sobre todo, prevenirla. Los comités de conservación de la vicuña han tomado a veces la decisión de dejar a los animales afectados de caspa para la campaña siguiente de esquila, sin considerar que este es un problema de gran envergadura socioeconómica para el sector..

El desconocimiento de cómo tratar esta enfermedad se traduce directamente en pérdidas económicas cuantiosas para el productor, pues no se sabe cuántos años demorará en caer la caspa, con lo que se recortaría la vida productiva de estos animales y se recortarían sensiblemente los ingresos, para no mencionar ya las dificultades que traería para el procesamiento de transformación primaria y el descerdado. Las pérdidas económicas que ocasiona la caspa son realmente considerable, pudiendo llegar a alrededor de 70 dólares americanos por vicuña (Informe del Proyecto Vicuñas: CC. CC. Lucanas-Ayacucho-2010).

\section{MATERIAL Y MÉTODO}

\section{Materiales}

\section{Lugar de estudio}

Se desarrolló en el Corral Perenne del Comité de Conservación y Uso Sostenible de la Vicuña Tullpacancha del Centro de Investigaciones, Producción y Transferencia Tecnológica Tullpacancha-UAP, ubicado en el distrito de Locroja y provincia de Churcampa, pertenecientes a la Región Huancavelica. Su altura oscila entre 3800 y 4500 m.s.n.m., y su temperatura promedio es de 5 C. Además, presenta temperaturas inferiores a $0{ }^{\circ} \mathrm{C}$ por las noches, con una humedad relativa de $67 \%$.

\section{Recurso}

Vicuña (Vicugna vicugna)

\section{Muestra de estudio}

La experiencia se desarrolló durante el III Chaco 2009 Tullpacancha - UAP, (19 de julio), donde para la toma de muestras del análisis visual se identificaron 74 vicuñas que presentaban anomalías de dermatitis escamosa o caspa, indistintamente del sexo, edad, zona afectada. La recolección de la muestra culminó durante el IV Chaco 2010 Tullpacancha - UAP (24 de agosto).

\section{Método de estudio}

\section{Primera etapa}

Colección de datos:

Los datos fueron tomados de animales con caspa evaluados en el momento de la esquila, según:

- Sexo de los animales

- Edad de las vicuñas

- Zona del cuerpo afectado.

Se identificaron a las vicuñas seleccionadas que presentaban caspa en diferentes partes del cuerpo. 
Tabla 2. Animales con caspa. Chaco 2009.

\begin{tabular}{|c|c|c|c|c|c|}
\hline & $\begin{array}{c}N^{\circ} \mathrm{DE} \\
\text { VICUÑAS }\end{array}$ & SEXO & EDAD & ÁMBITO & TRATAMIENTO \\
\hline 1 & 1 & $\mathrm{H}$ & A & ANCA & SÍ \\
\hline 2 & 26 & $\mathrm{H}$ & A & LOMO & Sí \\
\hline 3 & 12 & $\mathrm{H}$ & $\mathrm{A}$ & LOMO Y ANCA & Sí \\
\hline 4 & 1 & $\mathrm{H}$ & A & TODO & Sí \\
\hline 5 & 2 & $\mathrm{H}$ & $\mathrm{J}$ & ANCA & Sí \\
\hline 6 & 7 & $\mathrm{H}$ & $\mathrm{J}$ & LOMO & Sí \\
\hline 7 & 5 & $\mathrm{H}$ & $\mathrm{J}$ & LOMO Y ANCA & Sí \\
\hline 8 & 7 & $\mathrm{H}$ & $\mathrm{J}$ & TODO & Sí \\
\hline 9 & 7 & M & A & LOMO & Sí \\
\hline 10 & 3 & M & A & LOMO Y ANCA & Sí \\
\hline 11 & 1 & M & A & TODO & Sí \\
\hline 12 & 2 & M & $\mathrm{J}$ & TODO & Sí \\
\hline TOTAL & 74 & & & & \\
\hline
\end{tabular}

Fuente: Trabajo de campo. Población evaluada en la campaña III Chaco Tullpacancha 2009 que presentaba anomalías dermatológicas (caspa): 74 vicuñas, que constituía el 10,54\%

De acuerdo a la tabla anterior, se determina que la población afectada por la caspa alcanza una elevada proporción (10,54\%) en este corral perenne, y que la zona de mayor incidencia es la parte del lomo del animal: fibra A.

\section{Tratamiento curativo}

Todos los animales que presentaban cuadros con presencia de caspa (dermatitis escamosa) fueron sometidos a un tratamiento curativo veterinario, realizado de la manera siguiente:

Tabla 3. Tratamiento preventivo veterinario.

\begin{tabular}{c|c|c|}
\hline PROD. & PRINCIPIO & DOSIS \\
COMERCIAL & ACTIVO & $1,0 \mathrm{cc} /$ animal \\
\hline Ivomec & Ivermectina & $5,0 \mathrm{cc} /$ animal \\
\hline Hematovit & Vitaminas esenciales &
\end{tabular}




\section{Segunda etapa}

Tabla 4. Animales esquilados en el chaco 2010 que fueron tratados en el 2009.

\begin{tabular}{c|c|c|c|l|l|r} 
ARETE & SEXO & EDAD & $\begin{array}{c}\text { FIBRA } \\
(\mathrm{cm})\end{array}$ & \multicolumn{1}{|c}{ ÁMBITO } & PRESENCIA & $\begin{array}{c}\text { PESO } \\
(\text { gramos })\end{array}$ \\
\hline 303 & H & A & 2 & Inicio base & Lomo & 213 \\
270 & H & A & 2 & Inicio base & Lomo & 239 \\
\hline 311 & H & A & 2 & Medio & Lomo & 236 \\
301 & H & J & 2 & Al término & Lomo & 161 \\
\hline 286 & H & A & 2 & Medio & Lomo & 144 \\
91 & H & A & 2.5 & Medio & Lomo & 200 \\
\hline 307 & H & A & 2 & Medio & Lomo y anca & 238 \\
93 & H & A & 2 & Medio & Lomo y anca & 123 \\
276 & M & A & 2 & Medio & Lomo y anca & 202 \\
\hline 297 & H & A & 2 & Medio & Lomo y anca & 174 \\
\hline 269 & H & J & 2 & Inicio base & Lomo & 156 \\
\hline 302 & H & A & 1.5 & Medio & Lomo & 204 \\
\hline 287 & M & A & 2 & Medio & Lomo & 168 \\
\hline 296 & H & A & 2 & Medio & Lomo & 171 \\
\hline 289 & H & A & 2 & Medio & Lomo & 200 \\
\hline 15 & & \multicolumn{7}{c}{ TOTAL } & & 2,829 \\
\hline
\end{tabular}

Fuente: Registros de campo en el chacco 2010, en el CIPTT Tullpacancha.

\section{RESULTADOS}

Fueron los siguientes:

- Población evaluada en la campaña III Chaco Tullpacancha 2009 que presentó anomalías dermatológicas (caspa):

$$
74 \text { vicuñas }(100 \%)
$$

- Población recuperada: población que no presentó anomalías dermatológicas (caspa) en el IV Chaco 2010 Tullpacancha - UAP:

$$
15 \text { vicuñas }(20 \%)
$$

- Fibra recuperada que no presentó anomalías dermatológicas (caspa)en el IV Chaco 2010 Tullpacancha - UAP:

15 vicuñas $(2892 \mathrm{~g})$

\section{CONCLUSIONES}

$\mathrm{Al}$ analizar los datos obtenidos durante la presente investigación, se llegó a las siguientes conclusiones:

- De acuerdo con los cuadros anteriores, se determina que la población afectada con la caspa $(10,54 \%)$ en este corral perenne es alta, y que la zona de mayor incidencia es la parte del lomo del animal: fibra $\mathrm{A}$

- Las dosis de ivermectina y complejo vitamínico $\mathrm{A}, \mathrm{D}, \mathrm{E}, \mathrm{K}$ podrían tener una relación directa sobre los factores metabólicos, como causas posibles de la presencia de la dermatitis escamosa, por la disminución de la prevalencia. 
- Dado el número de muestras utilizado y la ausencia de 59 animales dosificados que no fueron capturados en el chaco 2010, no se puede aún definir plenamente las causas de la aparición de la dermatitis escamosa. Se está a la espera de una próxima evaluación.

\section{RECOMENDACIONES}

- Es importante continuar con una línea de investigación dirigida a la identificación de los agentes causales de la dermatitis escamosa (caspa), a fin de disminuir las pérdidas económicas que provoca esta afección a los vellones de fibra de vicuña.

- Es importante determinar los planes de manejo de la vicuña, con la finalidad de disminuir la incidencia de la caspa en los animales de los corrales perennes.

\section{REFERENCIAS BIBLIOGRÁFICAS}

1. Alzola, R. 2002. Guía de estudio: sistema tegumentario. Curso de histología, embriología $y$ teratología. pp. 3-12. UNCPBA. Facultad de Ciencias Veterinarias, Argentina.

2. Banks, W. 1998. Histologia veterinaria aplicada. 2da ed., p. 427-436. Editorial El Manual Moderno. México, 16 de marzo de 2010.

3. Brack, A. 2010. La vicuña. Perú Ecológico [en línea] 4(2). Consultado el 15 de febrero de 2010, en http://www. peruecologico.com.peleconeg_vicuna_masinfo. htm

4. Bustinza, V. 1986. Los camélidos sudamericanos y el desarrollo andino.
Universidad Nacional del Altiplano. Puno, Perú. 36pp.

5. J. Deza Rivasplata y L. Chacón. "Estudio de la capacidad de la biomasa forrajera accesible del CIPTT Tullpacancha, para determinar la densidad poblacional de la vicuña”. En: Ciencia y Desarrollo. Vol. 6 No 1/ Enerojunio 2005. Universidad Alas Peruanas.

6. CONACS. 2007. Ex-Consejo Nacional de Camélidos Sudamericanos. Boletín Informativo Institucional 2007. Ministerio de Agricultura, Lima, Perú.

7. Fowler, M. 1989. Medicine and Surgery of South American Camelids: Llama, Alpaca, Vicuña, Guanaco. 1ra ed., Ames, Iowa State University Press. pp. 176-179.

8. Gaitán, M. 1967. Estudio preliminar de los foliculos pilosos en alpacas variedad Huacaya. Tesis de ingeniero zootecnista. Facultad de Ingeniería Zootecnia, Universidad Nacional Agraria La Molina, Lima. 31 pp.

9. Santana, C. 1978. Estudio preliminar de la longitud y análisis cuticular en la fibra de la vicuña. Tesis de ingeniero zootecnista. Universidad Nacional Agraria La Molina, Lima, Perú. 166 pp.

10. Vila, B. 1989. La importancia de la etología en la conservación y manejo de la vicuña. Universidad Nacional de Luján, Luján, Argentina.

11. Wheeler, J. 1998. Diversidad genética y manejo de poblaciones de vicuña en el Perú. Visión Veterinaria, IVITA, Universidad Nacional Mayor de San Marcos, Lima, Perú.

12. Zúñiga Velando, Marco A. 2007. La vicuña y su manejo técnico. 1era. ed. Universidad Alas Peruanas, Perú, 166 pp. 
ANEXO 1

Fotografías

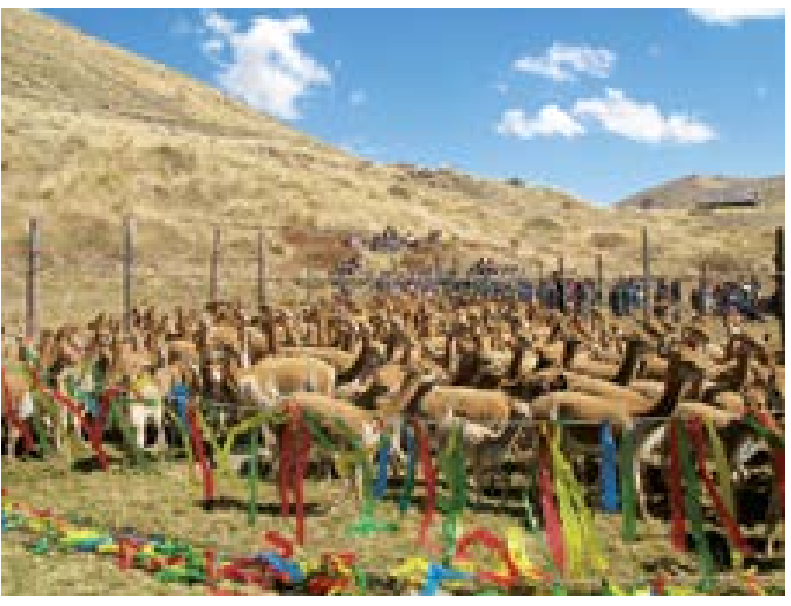

Figura 1. Chacco o captura de vicuñas.

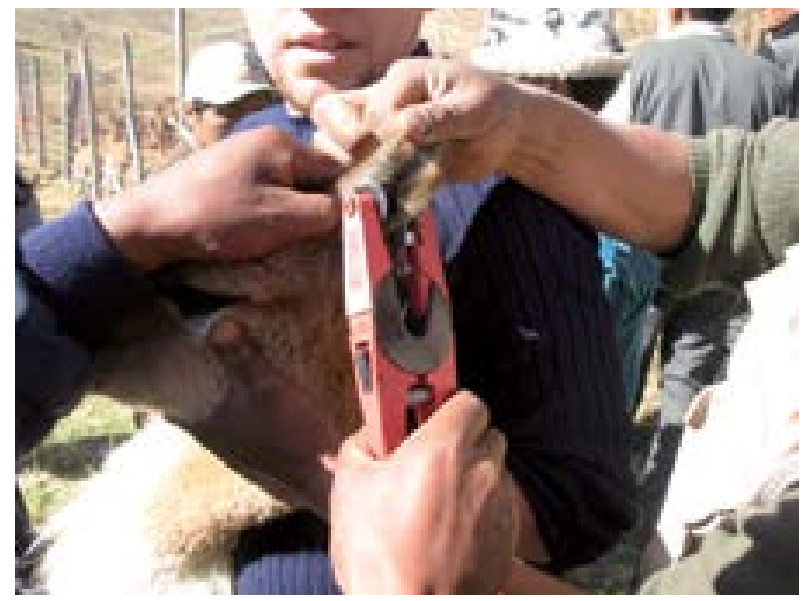

Figura 3. Aretado de vicuñas, que luego permitirá la identificación de cada individuo.

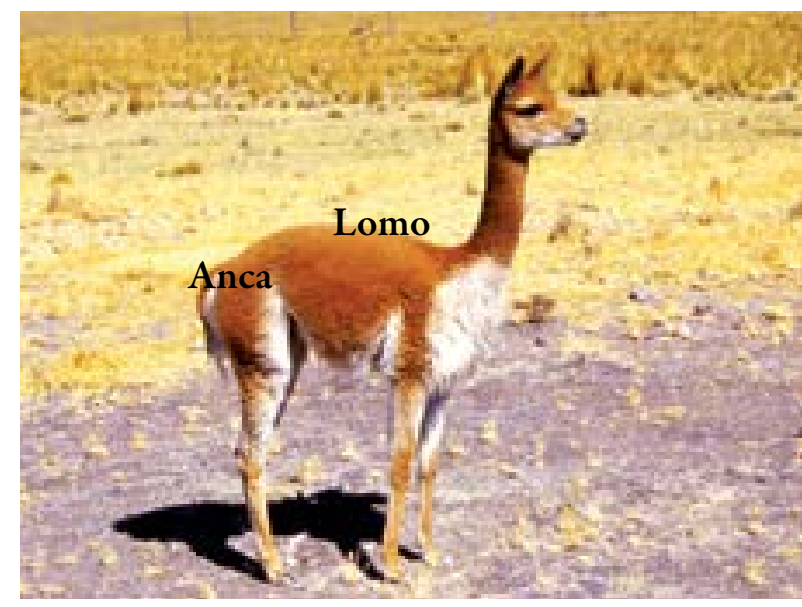

Figura 2. Zonas de identificación.

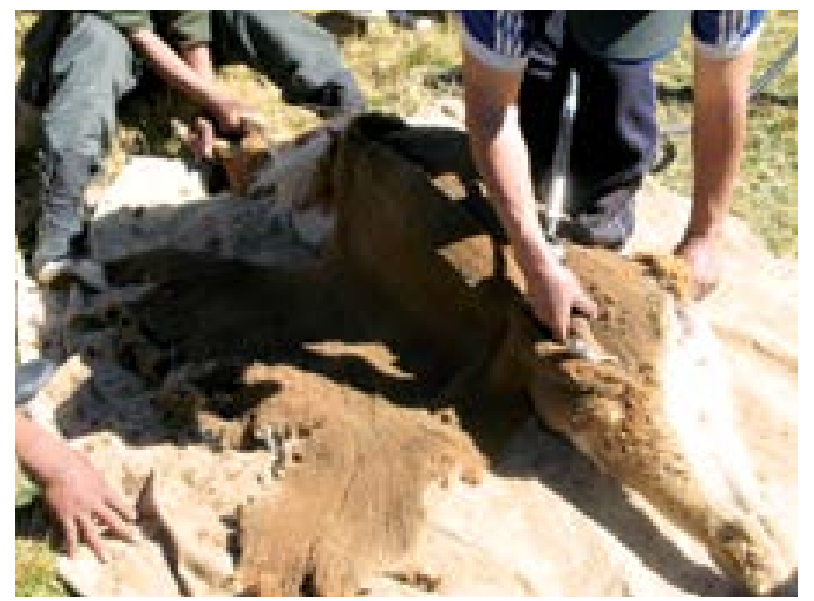

Figura 4. Proceso de esquila.

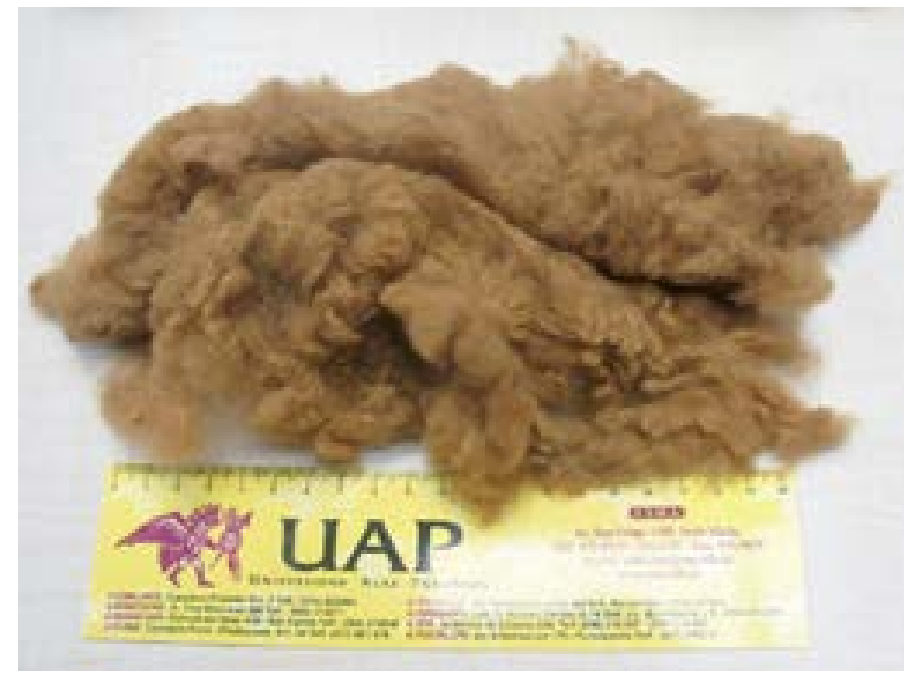

Figura 5. Fibra de vicuña. 


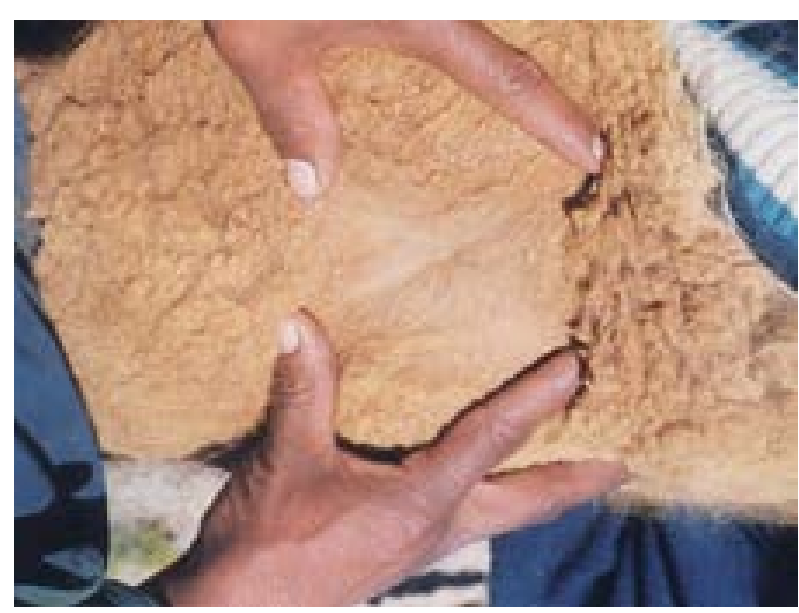

Figura 6. Caspa en la fibra de vicuña.

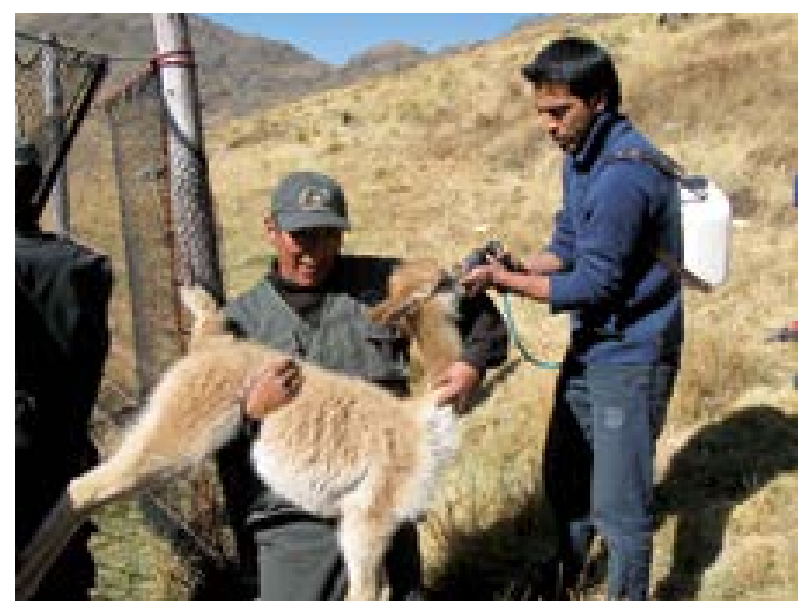

Figura 8. Suministro de vitamina B.

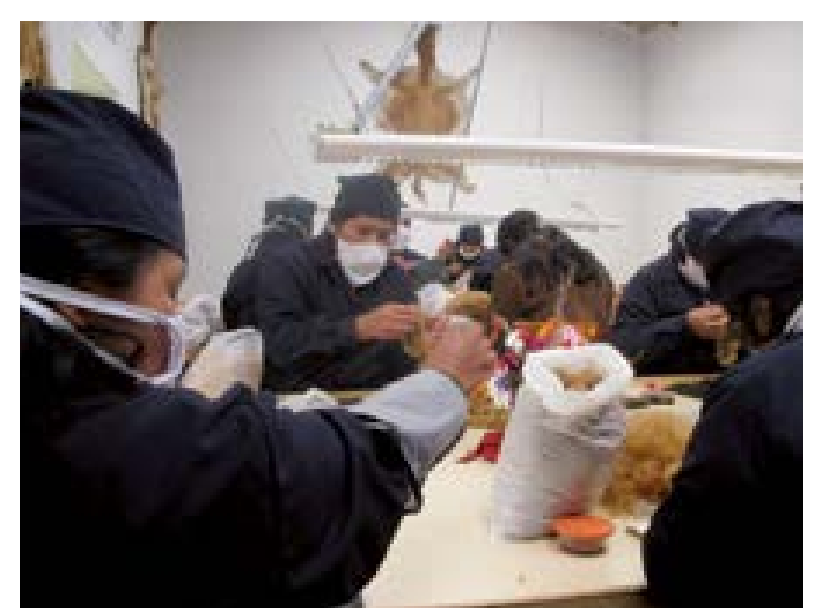

Figura 7. Taller de descerdado.

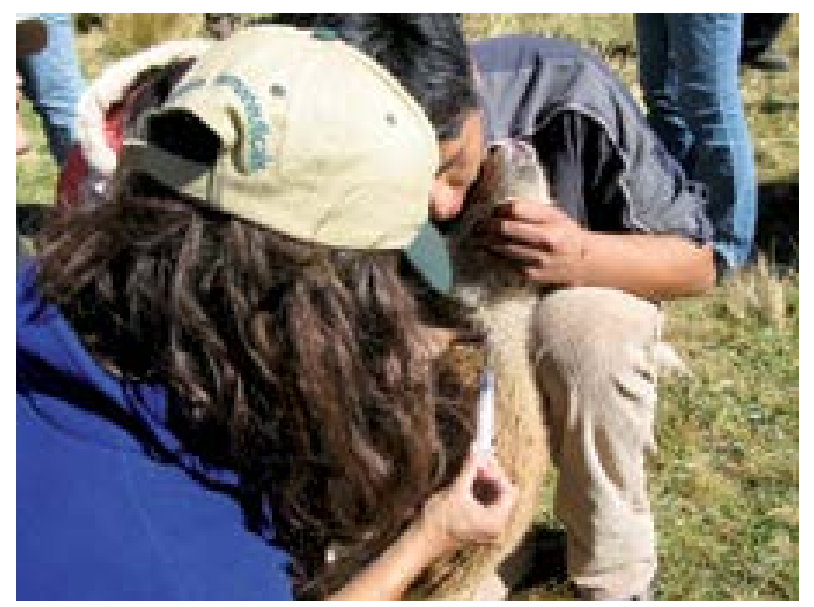

Figura 9. Aplicación de medicamentos.

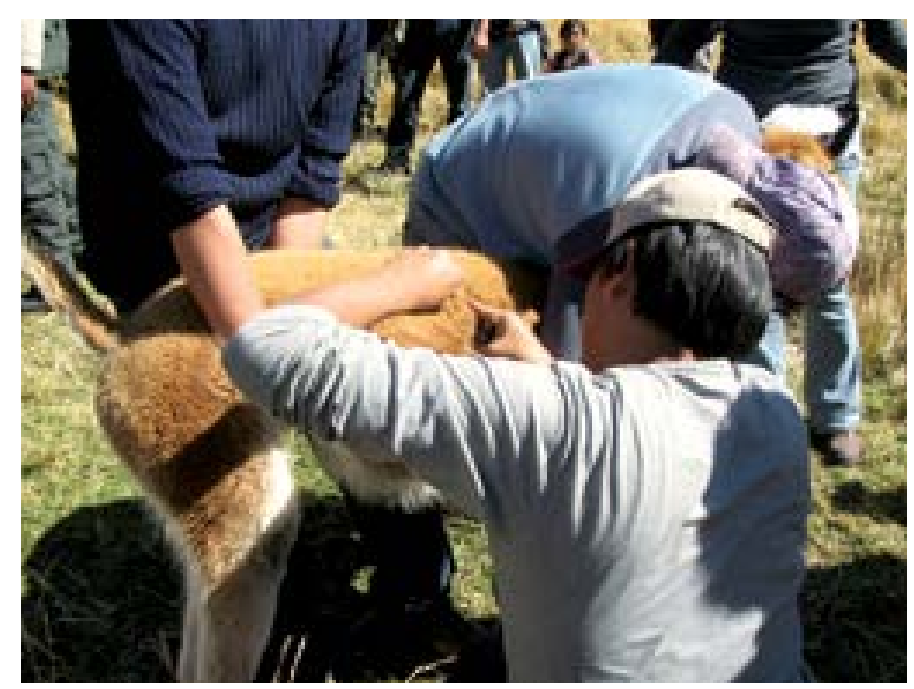

Figura 10. Evaluación e identificación de animales con dermatitis escamosa. 
ANEXO 2

Evaluación de animales con caspa

\begin{tabular}{|c|c|c|c|c|c|c|}
\hline \multicolumn{2}{|c|}{ ARETE } & SEXO & EDAD & ÁMBITO & FIBRA $(\mathrm{cm})$ & PRESENCIA \\
\hline 17.- & 281 & $\mathrm{H}$ & A & Anca & 2 & Medio \\
\hline & RETE & SEXO & EDAD & ÁMBITO & FIBRA $(\mathrm{cm})$ & PRESENCIA \\
\hline 3.- & 268 & $\mathrm{H}$ & A & Lomo & 1 & Medio \\
\hline 5.- & 270 & $\mathrm{H}$ & A & Lomo & 2 & Inicio base \\
\hline 7.- & 272 & $\mathrm{H}$ & A & Lomo & 2 & Medio \\
\hline 9.- & 274 & $\mathrm{H}$ & A & Lomo & 2 & Medio \\
\hline 13.- & 277 & $\mathrm{H}$ & A & Lomo & 2 & Medio \\
\hline & 279 & $\mathrm{H}$ & A & Lomo & 2 & Medio \\
\hline 20.- & 283 & $\mathrm{H}$ & A & Lomo & 1,5 & Inicio base \\
\hline 21.- & 284 & $\mathrm{H}$ & A & Lomo & 1,5 & Medio \\
\hline 23.- & 286 & $\mathrm{H}$ & A & Lomo & 2 & Medio \\
\hline 25.- & 288 & $\mathrm{H}$ & A & Lomo & 2 & Medio \\
\hline 26.- & 289 & $\mathrm{H}$ & A & Lomo & 2 & Medio \\
\hline 27.- & 290 & $\mathrm{H}$ & A & Lomo & 2 & Medio \\
\hline 34.- & 294 & $\mathrm{H}$ & A & Lomo & 2 & Inicio base \\
\hline 36.- & 296 & $\mathrm{H}$ & A & Lomo & 2 & Medio \\
\hline 40.- & 300 & $\mathrm{H}$ & A & Lomo & 2 & Medio \\
\hline 42.- & 302 & $\mathrm{H}$ & A & Lomo & 1,5 & Medio \\
\hline 43.- & 303 & $\mathrm{H}$ & A & Lomo & 2 & Inicio base \\
\hline 44.- & 304 & $\mathrm{H}$ & A & Lomo & 2 & Medio \\
\hline 46.- & 306 & $\mathrm{H}$ & A & Lomo & 2,2 & Inicio base \\
\hline 49.- & 309 & $\mathrm{H}$ & A & Lomo & 1,5 & Inicio base \\
\hline 51.- & 311 & $\mathrm{H}$ & A & Lomo & 2 & Medio \\
\hline 52.- & 312 & $\mathrm{H}$ & A & Lomo & 2 & Medio \\
\hline 54.- & 97 & $\mathrm{H}$ & A & Lomo & 2 & Medio \\
\hline 59.- & 90 & $\mathrm{H}$ & A & Lomo & 2 & Inicio base \\
\hline 60.- & 91 & $\mathrm{H}$ & A & Lomo & 2,5 & Medio \\
\hline 65.- & 96 & $\mathrm{H}$ & A & Lomo & 2 & Medio \\
\hline & RETE & SEXO & EDAD & ÁMBITO & FIBRA $(\mathrm{cm})$ & PRESENCIA \\
\hline 8.- & 273 & $\mathrm{H}$ & $\mathrm{A}$ & Lomo y anca & 2 & Medio \\
\hline 10.- & 275 & $\mathrm{H}$ & A & Lomo y anca & 2,5 & Medio \\
\hline 14.- & 278 & $\mathrm{H}$ & A & Lomo y anca & 2,2 & Medio \\
\hline 37.- & 297 & $\mathrm{H}$ & A & Lomo y anca & 2 & Medio \\
\hline 39.- & 299 & $\mathrm{H}$ & A & Lomo y anca & 2 & Medio \\
\hline 47.- & 307 & $\mathrm{H}$ & A & Lomo y anca & 2 & Medio \\
\hline 57.- & 88 & $\mathrm{H}$ & A & Lomo y anca & 1,5 & Medio \\
\hline 62.- & 93 & $\mathrm{H}$ & A & Lomo y anca & 2 & Medio \\
\hline
\end{tabular}




\begin{tabular}{|c|c|c|c|c|c|}
\hline 94 & $\mathrm{H}$ & A & Lomo y anca & 2,5 & Medio \\
\hline 64.- & $\mathrm{H}$ & A & Lomo y anca & 2 & Medio \\
\hline 68.- 99 & $\mathrm{H}$ & A & Lomo y anca & 2,5 & Medio \\
\hline 72.- 103 & $\mathrm{H}$ & A & Lomo y anca & 2,5 & Al término \\
\hline ARETE & SEXO & EDAD & ÁMBITO & FIBRA $(\mathrm{cm})$ & PRESENCIA \\
\hline 1.- $\quad 266$ & $\mathrm{H}$ & A & Todo & 2 & Medio \\
\hline ARETE & SEXO & EDAD & ÁMBITO & FIBRA $(\mathrm{cm})$ & PRESENCIA \\
\hline 70.- 101 & $\mathrm{H}$ & $\mathrm{J}$ & Anca & & Medio \\
\hline 74.- 105 & $\mathrm{H}$ & $\mathrm{J}$ & Anca & 2 & $\mathrm{Al}$ término \\
\hline ARETE & SEXO & EDAD & ÁMBITO & FIBRA $(\mathrm{cm})$ & PRESENCIA \\
\hline 4.- $\quad 269$ & $\mathrm{H}$ & J & Lomo & 2 & Inicio base \\
\hline 28.- $\quad 24$ & $\mathrm{H}$ & $\mathrm{J}$ & Lomo & 2 & Medio \\
\hline 33.- 293 & $\mathrm{H}$ & $\mathrm{J}$ & Lomo & 2 & Medio \\
\hline 41.- $\quad 301$ & $\mathrm{H}$ & $\mathrm{J}$ & Lomo & 2 & Al término \\
\hline 45.- $\quad 305$ & $\mathrm{H}$ & $\mathrm{J}$ & Lomo & 2,2 & Medio \\
\hline 58.- $\quad 89$ & $\mathrm{H}$ & $\mathrm{J}$ & Lomo & 1,5 & Medio \\
\hline 71.- 102 & $\mathrm{H}$ & $\mathrm{J}$ & Lomo & 2 & Inicio base \\
\hline ARETE & SEXO & EDAD & ÁMBITO & FIBRA $(\mathrm{cm})$ & PRESENCIA \\
\hline 38.- $\quad 298$ & $\mathrm{H}$ & $\mathrm{J}$ & Lomo y anca & 2 & Medio \\
\hline 50.- $\quad 310$ & $\mathrm{H}$ & $\mathrm{J}$ & Lomo yanca & 1,5 & Al término \\
\hline 53.- 716 & $\mathrm{H}$ & $\mathrm{J}$ & Lomo y anca & 1,5 & Medio \\
\hline 66.- & $\mathrm{H}$ & $\mathrm{J}$ & Lomo y anca & 2 & Medio \\
\hline 67.- 98 & $\mathrm{H}$ & $\mathrm{J}$ & Lomo y anca & 2 & Inicio base \\
\hline ARETE & SEXO & EDAD & ÁMBITO & FIBRA $(\mathrm{cm})$ & PRESENCIA \\
\hline 2.- $\quad 267$ & $\mathrm{H}$ & $\mathrm{J}$ & Todo & 2 & Medio \\
\hline 6.- $\quad 271$ & $\mathrm{H}$ & $\mathrm{J}$ & Todo & 2 & Al término \\
\hline 12.- 55 REP & $\mathrm{H}$ & $\mathrm{J}$ & Todo & 2 & Al término \\
\hline 16.- $\quad 280$ & $\mathrm{H}$ & $\mathrm{J}$ & Todo & 2 & Medio \\
\hline 18.- 122 & $\mathrm{H}$ & $\mathrm{J}$ & Todo & 2 & Inicio base \\
\hline 22.- $\quad 285$ & $\mathrm{H}$ & $\mathrm{J}$ & Todo & 2 & Medio \\
\hline 29.- 291 & $\mathrm{H}$ & $\mathrm{J}$ & Todo & 2 & Al término \\
\hline ARETE & SEXO & EDAD & ÁMBITO & FIBRA $(\mathrm{cm})$ & PRESENCIA \\
\hline 19.- 282 & M & A & Lomo & 2 & Medio \\
\hline 287 & M & A & Lomo & 2 & Medio \\
\hline 31.- & M & A & Lomo & 2 & Medio \\
\hline 32.- & M & A & Lomo & 2 & Medio \\
\hline 35.- 295 & M & A & Lomo & 2 & Inicio base \\
\hline 48.- $\quad 308$ & M & A & Lomo & 2 & Medio \\
\hline 56.- 87 & M & $\mathrm{A}$ & Lomo & 2 & Medio \\
\hline
\end{tabular}




\begin{tabular}{|c|c|c|c|c|c|}
\hline ARETE & SEXO & EDAD & ÁMBITO & FIBRA $(\mathrm{cm})$ & PRESENCIA \\
\hline 11.- 276 & M & $\mathrm{A}$ & Lomo y anca & 2 & Medio \\
\hline 55.- $\quad 42$ & M & A & Lomo y anca & 1,5 & Medio \\
\hline 61.- 92 & $\mathrm{M}$ & $\mathrm{A}$ & Lomo y anca & 2,5 & Medio \\
\hline ARETE & SEXO & EDAD & ÁMBITO & FIBRA $(\mathrm{cm})$ & PRESENCIA \\
\hline 69.- 100 & M & A & Todo & 3 & Medio \\
\hline ARETE & SEXO & EDAD & ÁMBITO & FIBRA $(\mathrm{cm})$ & PRESENCIA \\
\hline 30.- 292 & $\mathrm{M}$ & $\mathrm{J}$ & Todo & 2 & Medio \\
\hline 73.- 104 & M & $\mathrm{J}$ & Todo & 2 & $\mathrm{Al}$ término \\
\hline
\end{tabular}

